\title{
Subseasonal-to-seasonal predictability of the Southern Hemisphere eddy-driven jet during austral spring and early summer
}

Article

Accepted Version

Byrne, N. J., Shepherd, T. G. and Polichtchouk, I. (2019) Subseasonal-to-seasonal predictability of the Southern Hemisphere eddy-driven jet during austral spring and early summer. Journal of Geophysical Research: Atmospheres, 124 (13). pp. 6841-6855. ISSN 2169-8996 doi: https://doi.org/10.1029/2018JD030173 Available at https://centaur.reading.ac.uk/83703/

It is advisable to refer to the publisher's version if you intend to cite from the work. See Guidance on citing.

To link to this article DOI: http://dx.doi.org/10.1029/2018JD030173

Publisher: American Geophysical Union

All outputs in CentAUR are protected by Intellectual Property Rights law, including copyright law. Copyright and IPR is retained by the creators or other copyright holders. Terms and conditions for use of this material are defined in the End User Agreement. 


\section{CentAUR}

Central Archive at the University of Reading

Reading's research outputs online 


\title{
Subseasonal-to-seasonal predictability of the Southern Hemisphere eddy-driven jet during austral spring and early summer
}

\author{
Nicholas J. Byrne $^{1 *}$, Theodore G. Shepherd ${ }^{1}$, Inna Polichtchouk ${ }^{2}$ \\ ${ }^{1}$ Department of Meteorology, University of Reading, Reading, United Kingdom \\ ${ }^{2}$ European Centre for Medium-Range Weather Forecasts, Reading, United Kingdom
}

\section{Key Points:}

- SH midlatitude jet variations in spring and early summer are predictable several months ahead

- This subseasonal-to-seasonal predictability of the $\mathrm{SH}$ jet comes via the stratospheric polar vortex

- The observed influence of ENSO on the jet during this time is via this stratospheric pathway

*Present affiliation: European Centre for Medium-Range Weather Forecasts, Reading, UK

Corresponding author: Nicholas Byrne, n. byrne@ecmwf .int 


\section{Abstract}

Several recent studies have suggested that the stratosphere can be a source of subseasonalto-seasonal predictability of Southern Hemisphere circulation during the austral spring and early summer seasons, through its influence on the eddy-driven jet. We exploit the large sample size afforded by the hindcasts from the European Centre for Medium-Range Weather Forecasts Integrated Forecast System to address a number of unanswered questions. It is shown that the picture of coherent seasonal variability of the coupled stratospheretroposphere system apparent from the reanalysis record during the spring/early summer period is robust to sampling uncertainty, and that there is evidence of nonlinearity in the case of the most extreme variations. The effect of El Niño-Southern Oscillation on the eddy-driven jet during this time of year is found to occur via the stratosphere, with no evidence of a direct tropospheric pathway. A simple two-state statistical model of the stratospheric vortex is introduced to estimate the subseasonal-to-seasonal predictability associated with shifts of the seasonal cycle in the SH extratropical atmosphere. This simple model, along with a more general model, are subsequently used to interpret skill scores associated with hindcasts made using the full seasonal forecast model. Together the results provide evidence of tropospheric predictability on subseasonal-to-seasonal timescales from at least as early as August 1, and show no evidence of a 'signal-to-noise paradox' between the full seasonal forecast model and the reanalysis.

\section{Introduction}

Subseasonal-to-seasonal (S2S) forecasts for the extratropical troposphere are regularly regarded in a statistically heterogeneous manner; they are viewed as being most skilful during specific 'windows of opportunity' [WMO, 2013]. In this context, the influence of the stratosphere has received considerable attention in recent years. In the Northern Hemisphere (NH), winter is a period of particular focus. This is due to the occurrence of large perturbations to the stratospheric polar vortex (SPV), referred to as stratospheric sudden warmings (SSW), during the winter season. SSWs typically precede an equatorward shift of the tropospheric eddy-driven jet [EDJ; Baldwin and Dunkerton, 2001; Hitchcock and Simpson, 2014], and forecasts initialised during SSWs have been found to yield greater S2S forecast skill in the troposphere (in specific regions) than those that are not [Sigmond et al., 2013]. In the Southern Hemisphere (SH) SSWs are much rarer events [Roscoe et al., 2005], and interest has instead focused on the period in the lead- 
up to the annual SPV breakdown event, which generally occurs sometime in late spring/early summer [Black and McDaniel, 2007]. The strength of the SPV during this lead-up period has a strong influence on the timing of the breakdown event, as well as on the latitude of the EDJ in the troposphere [Byrne and Shepherd, 2018]. In addition, the SPV breakdown event itself typically precedes an equatorward shift of the EDJ [Byrne et al., 2017]. This close relationship between the SPV and the EDJ in the SH can be parsimoniously viewed as a continuous shift of the seasonal cycle during this time of year [Byrne and Shepherd, 2018]. This perspective suggests the potential for extended predictability in the extratropical SH troposphere during austral spring and summer, with the important caveat that there may be considerable sampling uncertainty associated with the magnitude of the predictable signal [Kumar, 2009]. Evidence for extended-range predictability during this time of year has been realised in a number of recent modelling studies [Roff et al., 2011; Son et al., 2013; Lim et al., 2013; Seviour et al., 2014], although most of these studies only considered sub-intervals of the entire spring/summer period and/or the observational record.

The phase of El Niño-Southern Oscillation (ENSO) offers another opportunity for extended range forecasts of the extratropical troposphere. In the SH, the observed extratropical response to ENSO shifts from a zonally asymmetric pattern in spring to a more zonally symmetric pattern in summer. This zonally symmetric pattern has been viewed as a forced response to ENSO via a direct tropospheric pathway [Seager et al., 2003; L'Heureux and Thompson, 2006; Lim et al., 2013]. However, the troposphere is not the only potential pathway for such remote extratropical impacts; the stratosphere provides another possible pathway. Work over recent decades has much improved the understanding of the relevant mechanisms for this stratospheric pathway [Domeisen et al., 2019]. Indeed, in regions such as the North Atlantic, impacts via the stratosphere are able to completely overwhelm any potential impacts via the troposphere [Polvani et al., 2017]. In the SH, the extratropical stratospheric pathway for ENSO is most prominent during austral spring and summer, a similar time period as for the observed zonally symmetric response to ENSO (Hurwitz et al. [2011]; Lin et al. [2012]; Zubiaurre and Calvo [2012]; see also Domeisen et al. [2019]). This poses the challenge of how best to separate the impacts of these two pathways, especially given the limited observational record. Byrne et al. [2017] attempted this separation in observations via a regression-based approach and concluded that the stratospheric pathway was dominant. More recent work 
by Vera and Osman [2018], who showed that the 'failed' zonally symmetric response to the large El Niño of 2015/2016 was consistent with an exceptionally strong SPV, also supports this conclusion.

In this paper we address these specific issues of S2S predictability and sampling variability for the $\mathrm{SH}$ extratropical atmosphere during the spring and summer period by analysing a large ensemble of seasonal forecast model data. We begin by using the ensemble to explore the impact of sampling uncertainty on some previous statistical results in the literature. We then use the ensemble to develop a simple statistical model for estimating the S2S predictability associated with shifts of the seasonal cycle in the SH extratropical troposphere. We subsequently use this simple model, along with the statistical model of Kumar [2009], to interpret skill scores associated with hindcasts made using the full seasonal forecast model. This includes investigation of whether there is any evidence of a mismatch between anomaly correlation and signal-to-noise ratio - known as the 'signal-to-noise paradox' [Scaife and Smith, 2018]. We conclude with a summary of our results.

\section{Data and Methods}

We use ECMWF System 4 hindcast data [Molteni et al., 2011]. System 4 is based on the IFS atmospheric component coupled to the NEMO ocean model. The atmospheric resolution is T255L91, which corresponds to approximately $80 \mathrm{~km}$ horizontally with 91 levels in the vertical. The resolution of the ocean model is 1 degree in the horizontal and has 42 layers in the vertical. All hindcasts are issued as ensembles with 51 members. The hindcast data is available over the period 1981-2016. Here we consider hindcasts initialised on August 1 and November 1 as these are the relevant dates for the period of austral spring and austral summer.

For verification we use the ERA-Interim reanalysis [Dee et al., 2011]. The basic data input for our study is daily-mean zonal wind and geopotential data for the period $1 \mathrm{Au}-$ gust 1981 to 31 January 2016, which encompasses 35 years in total. Data were available on a N128 Gaussian grid. Before analyzing the data, we first processed them by forming a zonal average. We denote this zonal average for the remainder of the paper using the [.] notation. We use zonally-averaged zonal wind $([\mathrm{u}])$ at $850 \mathrm{hPa}$ as a measure of the eddy-driven jet. We define a daily jet latitude index by computing the latitude of the 
maximum value of $[\mathrm{u}]$ between $35 \mathrm{~S}$ and $70 \mathrm{~S}$ at $850 \mathrm{hPa}$; no interpolation is used. We identify the date of the stratospheric vortex breakdown as the final time that $[\mathrm{u}]$ at $60 \mathrm{~S}$ drops below $10 \mathrm{~m} / \mathrm{s}$; we apply this criterion to running 5 -day averages at $50 \mathrm{hPa}$ [Black and McDaniel, 2007]. We define an index of interannual stratospheric variability as the leading principal component time series that emerges from a multiple empirical orthogonal function analysis on monthly-mean polar cap-averaged (60S - 90S) geopotential height at 50hPa, following Byrne and Shepherd [2018]. This method proceeds by combining X successive months of data in a vector for a given year, and then repeating this for all years. Each eigenvector will then have $\mathrm{X}$ elements. Here, we set $\mathrm{X}=6$, so as to span the entire austral spring and summer period. We define an ENSO index by averaging sea-surface temperatures across the Niño 3.4 region (5N-5S, 170W-120W). We define El Niño years as those years in the upper quartile of this index (i.e., the warmest $25 \%$ of years) and La Niña years as those years in the lower quartile (i.e., the coldest $25 \%$ of years). We examine the sea ice evolution using monthly-mean sea ice extent data from the U.S. National Snow and Ice Data Center (www.nsidc.org).

\section{Role of Sampling Uncertainty}

We begin by comparing the large-scale extratropical circulation during austral spring and summer in the hindcasts and in ERA-Interim. The purpose of this is two-fold. Firstly, we wish to confirm that the hindcasts have realistic circulation statistics. Secondly, once that is confirmed, the large hindcast ensemble size allows us to explore the potential impact of sampling uncertainty on the reanalysis results, to determine their robustness as well as to explore possible nonlinearities. Thus, the comparison between hindcasts and observations works in both directions. In most of what follows we exclude the year of 2002 from our analysis. The only SSW in the SH in the observational record occurred in 2002 [Roscoe et al., 2005], an event which was notable for its extreme impacts in both the stratosphere and troposphere [Thompson et al., 2005]. We exclude 2002 so that our results are not unduly reliant on such an extreme event.

\subsection{The eddy-driven jet}

Figure 1a shows the long-term average for the EDJ in ERA-Interim and Figure 1b shows a similar quantity for the hindcasts, based on initialisations on August 1. The semiannual oscillation [SAO; van Loon, 1967] in the latitude of the EDJ is visible in both pan- 
(a) ERA-Interim

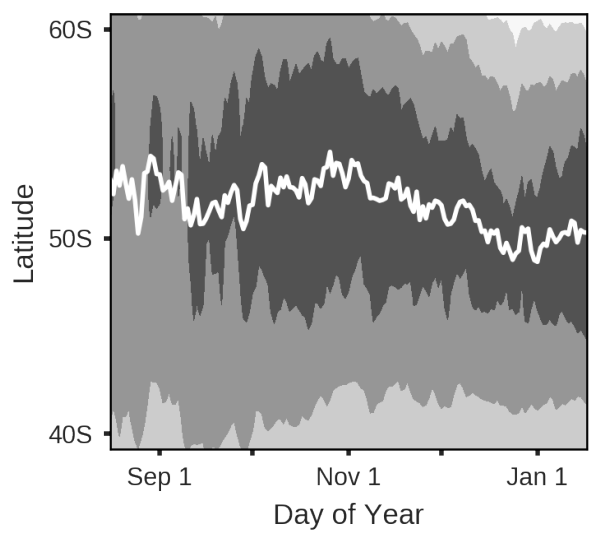

(b) Hindcasts

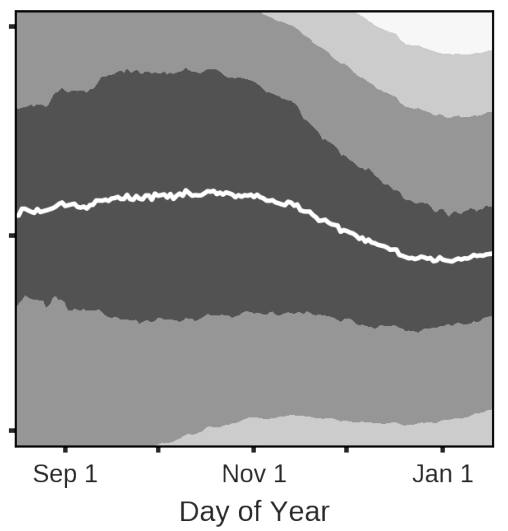

Figure 1. (a) Climatology of $[\mathrm{u}] 850 \mathrm{hPa}(\mathrm{m} / \mathrm{s}$, shading) and jet-latitude index (white line) for ERA-Interim, 1981 - 2015. The year of 2002 has been excluded. (b) Similar, but for the entire hindcast ensemble based on August 1 initialisations.

els from about October onwards, with the EDJ seen to be closer to the pole in spring and closer to the equator in summer. The impact of sampling uncertainty is visible in the 'noisy' appearance of the ERA-Interim average, and also in the relatively large confidence intervals for such averages during spring and early summer (Figure 2). There is a suggestion of an equatorward model bias developing several months into the hindcast, with this bias becoming gradually more noticeable as the summer months progress. However, even six months after the model initialisation, the magnitude of this potential bias is still within the sampling uncertainty (Figure 2). The hindcasts also appear to mimic observed variability in the EDJ, with a noticeable seasonal decrease in the variability of the latitude of the EDJ in both the hindcasts and the observations (Figure 3). Figure 4 provides a broader assessment of model zonal-wind bias for the August 1 initialisations. Broadly speaking, outside of the tropics and some very high-latitude regions during the month of September, there appears to be good agreement between the hindcasts and ERAInterim in the stratosphere. In the troposphere, an equatorward bias in the EDJ is seen to emerge from about December onwards, consistent with what was noted for Figure 2. 


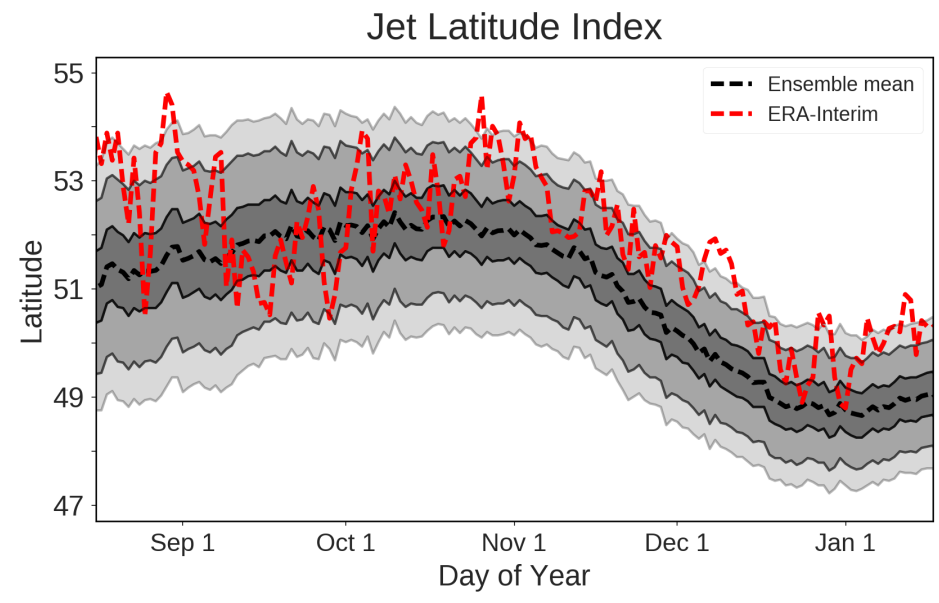

Figure 2. Bootstrap estimate of sampling uncertainty associated with 34-year mean of jet latitude using ensemble members from the August 1 initialisations. The bootstrap estimate was generated using 10000 time series of length 34 where an ensemble member has been randomly selected from each year in the 34-year period excluding the year of 2002. Solid lines represent 1, 5, 25, 75, 95 and 99\% thresholds respectively. Dashed red line indicates jet latitude from ERAInterim.

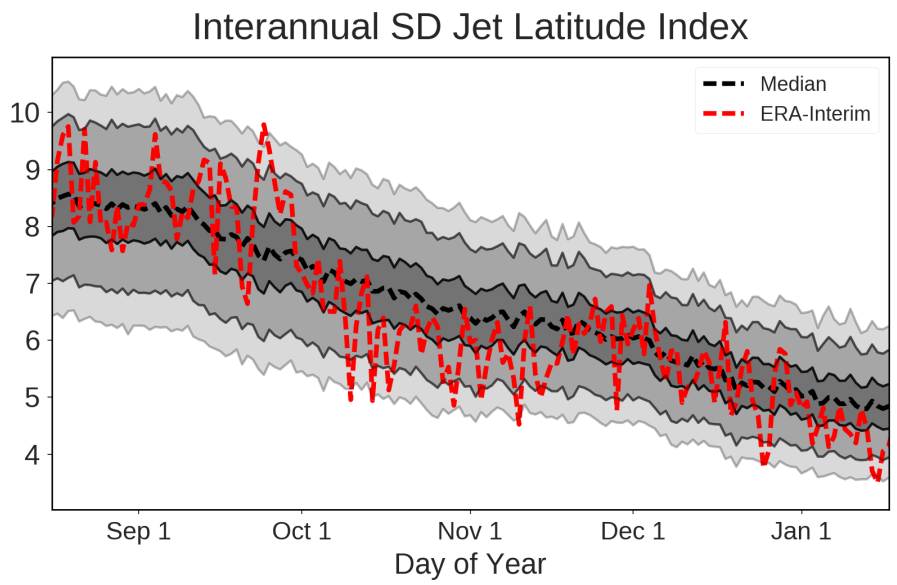

Figure 3. Similar to Figure 2, except using standard deviation instead of mean. 
(a) August (m/s)

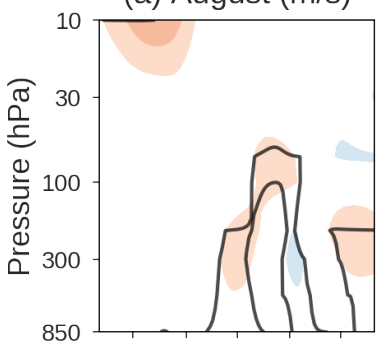

(d) November $(\mathrm{m} / \mathrm{s})$

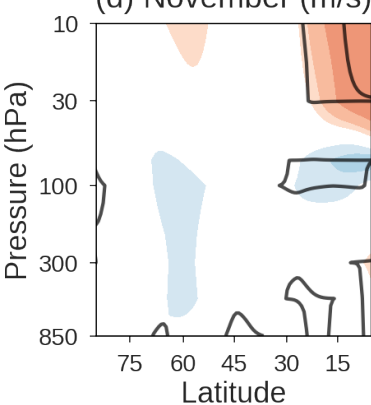

(b) September $(\mathrm{m} / \mathrm{s})$

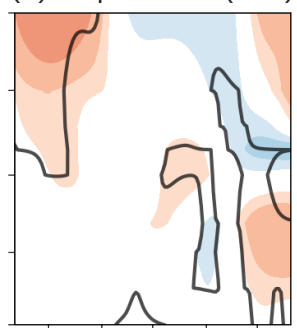

(e) December $(\mathrm{m} / \mathrm{s})$

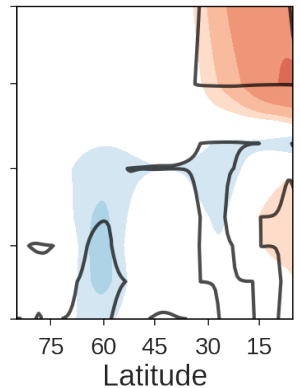

(c) October $(\mathrm{m} / \mathrm{s})$

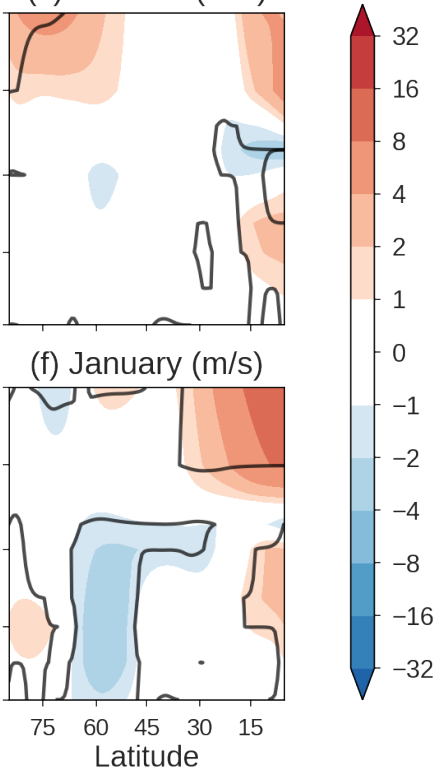

Figure 4. Monthly-mean climatological differences in $[\mathrm{u}]$ between hindcasts and ERA-Interim (m/s, shading) for (a) August, (b) September, (c) October, (d) November, (e) December and (f) January, 1981-2015. The year of 2002 has been excluded. Black contours indicate differences that are statistically different at the $1 \%$ level based on a two-sided two-sample t-test. Hindcasts initialised on August 1. 
(a) August (m/s)

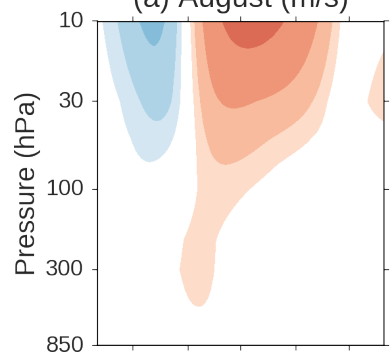

(d) November $(\mathrm{m} / \mathrm{s})$

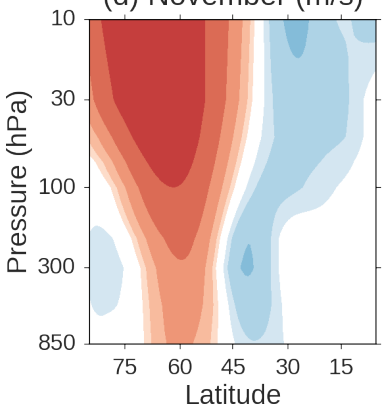

(b) September $(\mathrm{m} / \mathrm{s})$

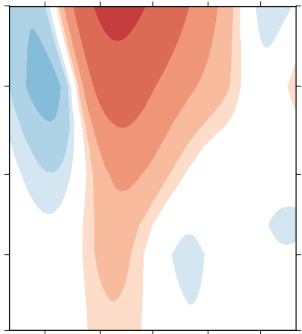

(e) December $(\mathrm{m} / \mathrm{s})$

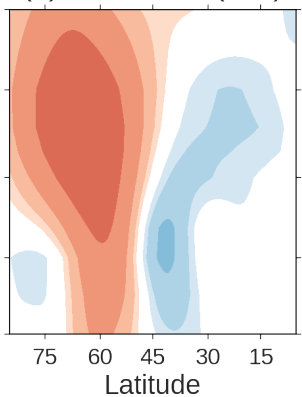

(c) October $(\mathrm{m} / \mathrm{s})$

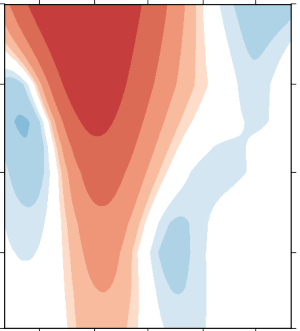

(f) January (m/s)

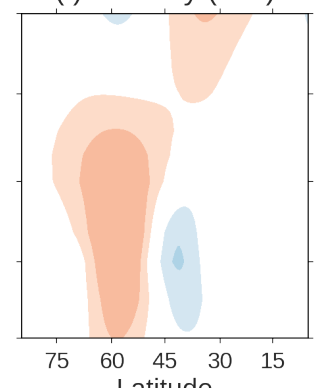

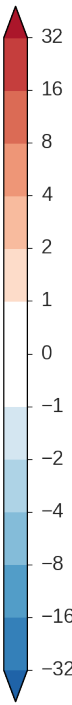

4

2

1

Figure 5. Monthly mean differences in $[\mathrm{u}]$ between upper and lower quartiles of the hindcast ensemble for August 1 initialisations, based on an index of stratospheric variability (see text for details). Please note that Figure 5 from Byrne and Shepherd [2018] represents the difference between upper and lower halves of the data, rather than the upper and lower quartiles used here, because of the limited sample size of the observational record. The difference between the upper and lower halves of the data for the hindcast ensemble is included in the Supplementary Material. Please also note the nonlinear color scale that is required for including tropospheric and stratospheric differences in the same figure. The year of 2002 has been excluded from all calculations.

This equatorward bias in December and January is not present in hindcasts initialised on November 1 (see Supplementary Material). To confirm that our results are not unduly sensitive to model bias, we verify that all results obtained for December and January are robust to the choice of initialisation date.

Next, we move on to considering coupled variability between the SPV and the EDJ. Figure 5 represents an estimate of this variability using the hindcast ensemble. It was constructed in a similar manner to previous reanalysis-based results ([Byrne and Shepherd, 2018]; see also Hio and Yoden [2005]). Briefly, an index of interannual variability in the extratropical stratosphere was applied to individual years in the ensemble. This 
index was then used to stratify the ensemble into quartiles, and to produce a composite difference between the lower and upper quartiles. The coupled patterns that are evident in Figure 5 can be parsimoniously viewed as a continuous shift of the seasonal cycle in the stratosphere and the troposphere during this time of year [Byrne and Shepherd, 2018]. During years where the stratospheric seasonal cycle is delayed in spring, there tends to be a corresponding delay in the timing of the SPV breakdown event in early summer; such years also tend to be associated with a stronger poleward shift of the EDJ between September-November and with a delay in the equatorward shift of the EDJ in early summer. The converse behaviour is found to occur on average in years with an accelerated stratospheric seasonal evolution in spring. The patterns of coupled stratospheretroposphere variability seen in Figure 5 are very similar to those found for reanalyses ([Byrne and Shepherd, 2018]; see also Hio and Yoden [2005]). The large ensemble size used here ensures a high degree of statistical confidence in all plotted differences, indicating that previous reanalysis-based results using 38 years of data are qualitatively robust to sampling variations.

Figure 6 focuses on the equatorward transition of the EDJ in early summer using hindcasts initialised on November 1 , so that the hindcasts are as close to observations as possible during the vortex breakdown period. The timing of the equatorward transition of the EDJ has been found to be closely coupled to the SPV breakdown date in the reanalysis [Byrne et al., 2017]. In particular, years with a later than average SPV breakdown date are associated with a later than average equatorward transition of the EDJ, with opposite behaviour for earlier than average years. To test the robustness of this relationship to sampling variability, we first define an index for the SPV breakdown date [Black and McDaniel, 2007] and apply this index to all years in the ensemble. We then divide the ensemble into late (L; upper half) and early (E; lower half) and plot daily averages of the EDJ (Figures 6a and 6b); both late and early sets contain approximately 900 breakdown events. These figures confirm what was previously found for the reanalysis: earlier SPV breakdown years are seen to have an earlier equatorward transition of the EDJ, with opposite behaviour in late SPV breakdown years. This behaviour can be seen most clearly in Figure 6c, where the difference between late and early years is shown.

We can also exploit the large ensemble size to explore the extremes of the system behaviour. One motivation for studying circulation extremes at this time of year is that they may be relevant for reducing the uncertainty in the ozone-hole-induced tropospheric 
(a) L Years

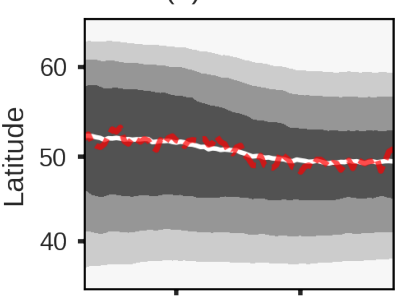

(d) LX Years

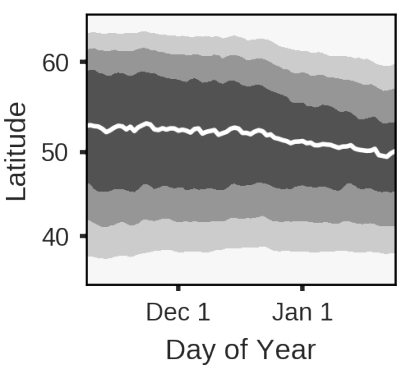

(b) E Years

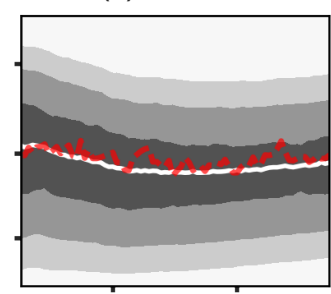

(e) EX Years

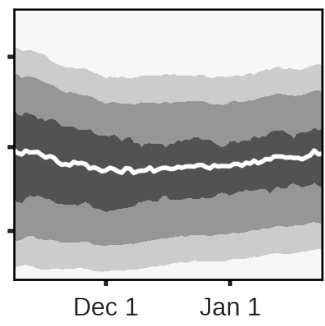

Day of Year (c) Difference

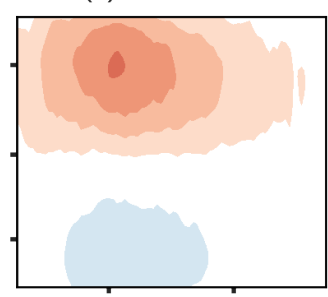

15

12

9

6

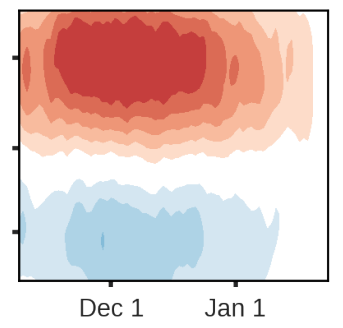

Day of Year

Figure 6. Average of $[\mathrm{u}] 850 \mathrm{hPa}(\mathrm{m} / \mathrm{s}$, shading) and jet-latitude index (white line) for (a) upper half (late), (b) lower half (early), (d) upper decile (extreme late) and (e) lower decile (extreme early) of SPV breakdown years in hindcast ensemble (see text for further details). Panel (c) shows difference between (a) and (b) and panel (f) shows difference between (d) and (e). The contour interval in (c) and (f) is $1 \mathrm{~m} / \mathrm{s}$, with values between -1 and $1 \mathrm{~m} / \mathrm{s}$ set to white. The red dashed line indicates jet-latitude index for (a) upper half (late) and (b) lower half (early) of SPV breakdown years from ERA-Interim (see also Byrne et al. [2017]). The year of 2002 has been excluded from all calculations. 
circulation changes [see Son et al., 2018, and references therein]. Here we define extreme late (LX) and early (EX) SPV breakdown years as upper and lower deciles of the data (Figure 6d and Figure 6e). There is a qualitative similarity in the behaviour between L and LX years, and similarly for E and EX years, with the timing of the equatorward transition of the EDJ seen to shift with the timing of the SPV breakdown date. However, it is also clear that this transition appears to proceed substantially less equatorward in LX years compared to EX years, with the result that perturbations to EDJ latitude appear to persist well into January in LX years (Figure 6f). Thus, a model of circulation variability that accounts for both a shift in timing and change in amplitude of the EDJ transition would appear most appropriate for characterising long-term changes. Such a model was previously proposed by Sun et al. [2014] as an explanation for the recent trends in the troposphere and stratosphere; the results of the hindcast ensemble used here would appear to lend further support to this hypothesis.

\subsection{ENSO}

The observed zonally symmetric extratropical summertime response to ENSO is characterised as a shift in latitude of the EDJ [Seager et al., 2003; L'Heureux and Thompson, 2006; Lim et al., 2013]. One complication in using a reanalysis to quantify the magnitude of this effect is that the limited observational record makes it difficult to control for potentially confounding effects such as the SPV, i.e. to distinguish between tropospheric and stratospheric pathways. Here we try to overcome this difficulty by exploiting the large ensemble size. Formally, given a variable X (EDJ) that is potentially responsive to variables $\mathrm{Y}$ (ENSO) and Z (SPV), we consider the difference in $\mathrm{X}$ between two extremes of $\mathrm{Y}$ while holding $\mathrm{Z}$ fixed, and similarly for extremes of $\mathrm{Z}$ while holding Y fixed.

To begin, we address the reverse question of whether the influence of the SPV on the EDJ might be confounded by the influence of ENSO. To do this, we perform a similar analysis to Figure 5 but only allowing years where an El Niño event was simulated in the hindcasts i.e., we condition on El Niño events (Figure 7). The results are virtually identical to Figure 5. This shows that the results from the previous section are robust to potentially confounding effects from ENSO. We now proceed to explore the EDJ response to ENSO in the hindcast ensemble. Figure 8 shows the El Niño minus La Niña response for hindcasts initialised on August 1. Consistent with the previously mentioned 
(a) August (m/s)

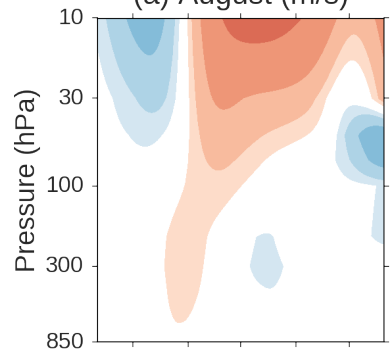

(d) November $(\mathrm{m} / \mathrm{s})$

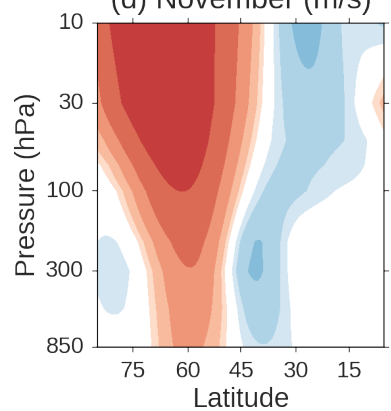

(b) September $(\mathrm{m} / \mathrm{s})$

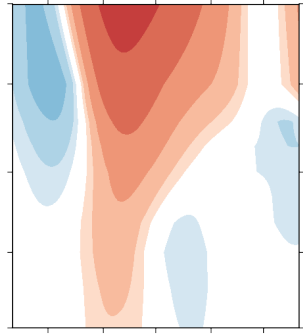

(e) December $(\mathrm{m} / \mathrm{s})$

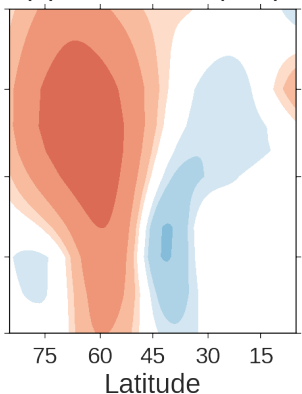

(c) October $(\mathrm{m} / \mathrm{s})$
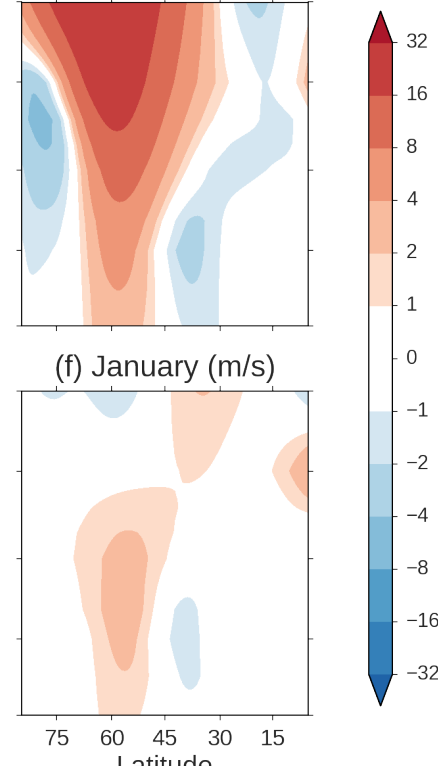

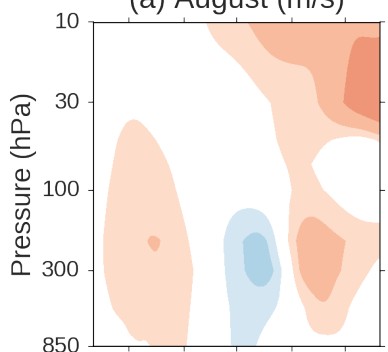

(d) November $(\mathrm{m} / \mathrm{s})$

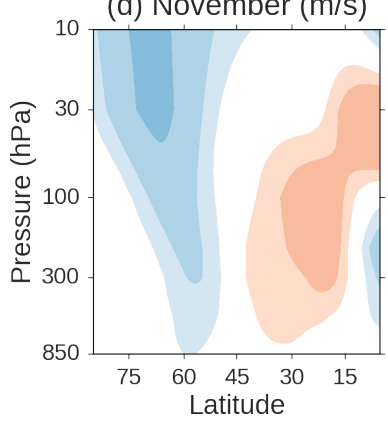

(b) September $(\mathrm{m} / \mathrm{s})$

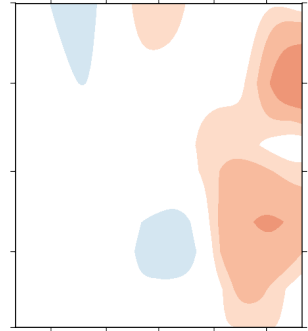

(e) December (m/s)

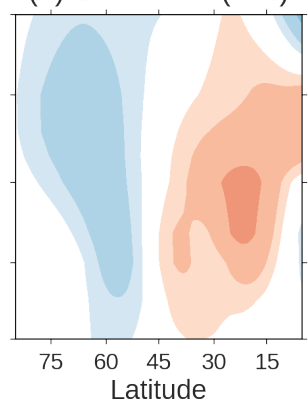

(c) October $(\mathrm{m} / \mathrm{s})$

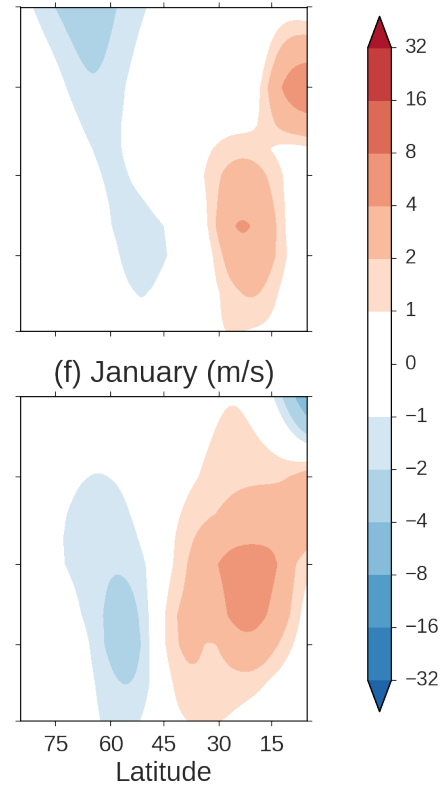

Figure 8. Monthly-mean differences in $[\mathrm{u}]$ between El Niño and La Niña years for August 1 initialisation (see text for details). The year of 2002 has been excluded from all calculations. 
(a) August (m/s)

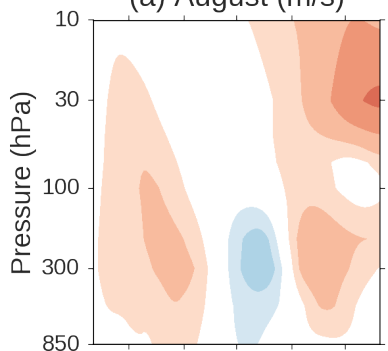

(d) November $(\mathrm{m} / \mathrm{s})$

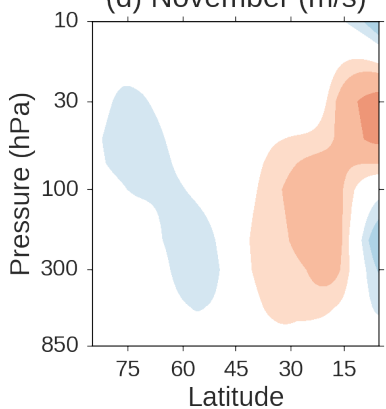

(b) September $(\mathrm{m} / \mathrm{s})$

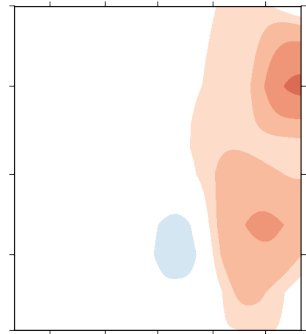

(e) December $(\mathrm{m} / \mathrm{s})$

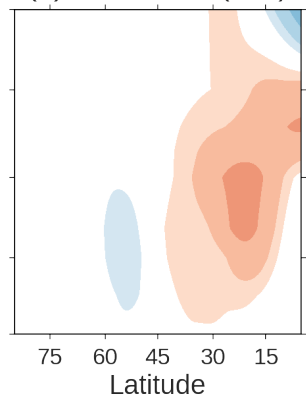

(c) October $(\mathrm{m} / \mathrm{s})$

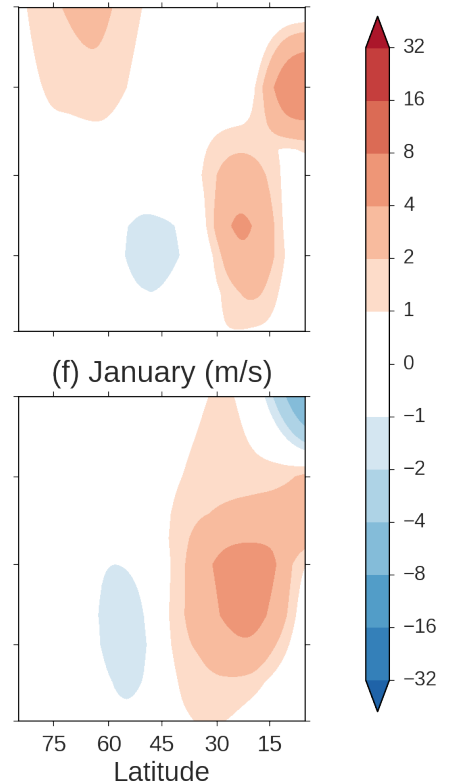

Figure 9. Similar to Figure 8, but conditioned on lower quartile of stratospheric variability index (see text for details). The year of 2002 has been excluded from all calculations. See Supplementary Material for differences conditioned on upper quartile of stratospheric variability index.

results for the reanalysis, an EDJ response is seen to emerge from about November onwards. However, this figure also contains evidence of an SPV response to ENSO that precedes the EDJ response in time. This suggests that the SPV may be acting as a confounding variable or, equivalently, that any potential ENSO-EDJ link is via a stratospheric pathway. To test this hypothesis, we repeat our analysis using only years from the lower quartile of our stratospheric variability index i.e., we condition on the SPV (Figure 9). The large reduction in the EDJ response in this figure is consistent with our hypothesis of a stratospheric pathway for the ENSO-EDJ influence, in that the influence is markedly reduced when the stratospheric pathway is blocked by conditioning on the SPV.

As a complementary approach to this conditional analysis, we also perform a regressionbased analysis similar to that described in L'Heureux and Thompson [2006] and Byrne et al. [2017]. Briefly, monthly-mean [u] at $850 \mathrm{hPa}$ and averaged over $55-65 \mathrm{~S}$ is correlated against an index for ENSO for November, December and January separately. This analysis is then repeated after first linearly regressing out the impact from the SPV (see Byrne 


\begin{tabular}{||ccccc|}
\hline Month & ERA & ERA - No SPV & Hindcasts & Hindcasts - No SPV \\
\hline \hline Nov & 0.26 & 0.14 & $0.14(-0.14,0.40)$ & $0.08(-0.21,0.35)$ \\
\hline Dec & $\mathbf{0 . 3 3}$ & 0.14 & $0.19(-0.08,0.44)$ & $0.07(-0.20,0.34)$ \\
\hline Jan & 0.16 & 0.01 & $0.24(-0.03,0.48)$ & $0.03(-0.24,0.29)$ \\
\hline
\end{tabular}

Table 1. Correlation between $850 \mathrm{hPa}[\mathrm{u}]$ averaged over 55-65S and ENSO, for ERA-Interim and for hindcasts based on August 1 initialisation, with and without the influence from the SPV. Hindcast columns show median value along with 5 and $95 \%$ confidence intervals. See text for further details. Bold values indicate quantities that are statistically different from zero at the $5 \%$ level based on a one-sided two-sample t-test.

et al. [2017] for further details). The results from ERA-Interim are shown in Table 1. They indicate that the correlation between ENSO and EDJ is relatively weak, and that it is further reduced once the stratospheric pathway has been removed. To compare these observational results against the hindcasts, we begin by generating a synthetic time series of length 34 by randomly selecting an ensemble member from each year (excluding 2002) for hindcasts initialised on August 1. We then repeat the above correlation analysis for this synthetic time series. We do this for 10000 synthetic time series to generate a distribution. The results are again shown in Table 1. Firstly, it is clear that all values from observations lie within the 5 and $95 \%$ confidence intervals for the hindcasts. This indicates that any differences in the correlations between the observations and the hindcasts are consistent with sampling variability. Secondly, the results from the hindcasts indicate that any correlation between ENSO and EDJ essentially vanishes once the stratospheric pathway has been controlled for. Thus, combining these results with the previous results from the conditional analysis, we conclude that there is a close relationship between the SPV and EDJ throughout austral spring and summer, and this relationship means that the SPV has the potential to act as a confounding variable unless suitably controlled for. It should be noted that although our results suggest that any tropospheric ENSO-EDJ pathway is weak through spring and early summer, they do not preclude the existence of a tropospheric pathway following the breakdown of the SPV. Further research is required to establish the robustness of any such pathway. 


\section{S2S Hindcasts of the Extratropical Circulation}

In the previous section a robust relationship was established between perturbations to the seasonal cycle of the SPV and the seasonal cycle of the EDJ from August until January. In addition, evidence was presented that any potential relationship between ENSO and the EDJ during this time period was also likely via (perturbations to the seasonal cycle of) the SPV. A natural question that emerges from these results is whether such shifts of the seasonal cycle are predictable on S2S timescales, and if so, whether they might allow skilful forecasts of the EDJ on S2S timescales?

To begin to answer this question, firstly we note that several previous studies have highlighted that the SH SPV exhibits long autocorrelation timescales from August until December [see Gerber et al., 2010, and references therein]. These long timescales can be viewed as evidence for the predictability of shifts of the seasonal cycle of the SPV. Indeed, such predictability has been found for hindcasts from August 1 in a previous study [Seviour et al., 2014]. We reach similar conclusions for the hindcasts from August 1 that are used in this study by computing receiver operating characteristic (ROC) curves for early, late, extreme early and extreme late years for the SPV in observations (Figure 10). Here we have repeated our analysis from the previous section and classified each year from the observations as early, late, extreme early or extreme late depending on whether it is contained in the lower or upper halves or quartiles of the index of interannual stratospheric variability in observations. The ROC curves indicate that the model appears able to predict shifts of the seasonal cycle based on an August 1 initialisation, with a suggestion of greater forecast skill for years with a more extreme shift of the seasonal cycle.

Given these apparently predictable shifts of the seasonal cycle of the SPV, we now explore the implications for S2S forecasts of the EDJ. We do this in three ways. First, we introduce a simplified two-state model in an attempt to better understand the 'signal' and 'noise' characteristics of the full system. We then compare the predictions of this simplified model against those that emerge from a more general model of signal and noise [Kumar, 2009]; this model is more general as it permits a continuous rather than a discrete (i.e., two-state) representation of the signal. Finally, we compare both of these results against estimates of skill derived from verifying hindcast data against observations. It is assumed that conclusions that are common to all three methods will not be unduly sensitive to the underlying assumptions for any one particular method. 


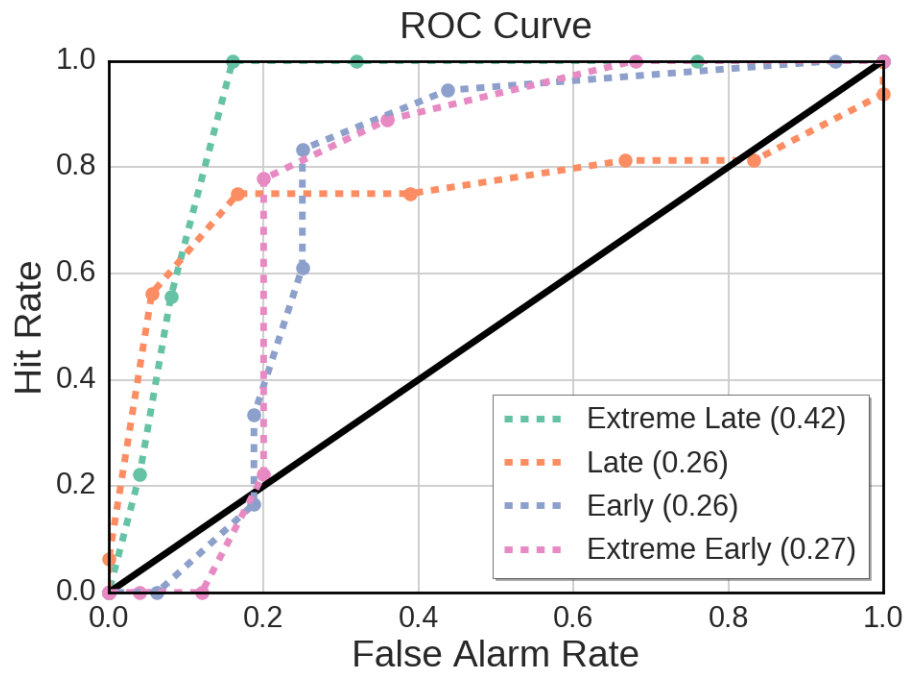

Figure 10. ROC curves for hindcasts for extreme late, late, early and extreme early stratosphere years from ERA-Interim (see text for details on how these years are defined). Values in brackets indicate area under ROC curves minus area under black diagonal line. The year of 2002 has been excluded from all calculations.

To derive our simplified two-state model we begin by assuming that individual years can be classified into one of two types, late or early, and that both types occur with equal probability (here late and early refer to upper and lower halves of the index of stratospheric interannual variability). We stress that this is a gross simplification of the actual system behaviour, where shifts of the seasonal cycle are likely better viewed as a continuous spectrum rather than as discrete regimes. Next we assume that these two types of years can be characterised by their means $\left(\mu_{L}, \mu_{E}\right)$ and standard deviations $\left(\sigma_{L}, \sigma_{E}\right)$, and that $\sigma_{L}=\sigma_{E}$. We use the large model ensemble to estimate values for all of these quantities (Figure 11); it should be noted that all of these quantities are a function of calendar day. Finally, we assume that for each year our model is able to forecast whether a late or early year will occur, but nothing further. This means that for each individual year, our ensemble-mean forecast will be $\left(\frac{\mu_{L}+\mu_{E}}{2} \pm \frac{\mu_{L}-\mu_{E}}{2}\right)$. For a sufficiently large ensemble and verification time series, we expect that forecast skill should be a function of a so-called 'signal-to-noise' ratio $\frac{\mu_{L}-\mu_{E}}{2 \sigma_{L}}$ (see Appendix A of Kumar [2009] for a derivation of how this ratio can be related to forecast skill). In what follows we use anomaly correlation $(\mathrm{AC})$ as our measure of forecast skill; the expected value of $\mathrm{AC}$ is equal to $\frac{s}{\left(1+s^{2}\right)^{0.5}}$, where $s=\frac{\mu_{L}-\mu_{E}}{2 \sigma_{L}}$. For later sections it is helpful to remember that, even for 
(a) $50 \mathrm{hPa}[\mathrm{u}] 55-65 \mathrm{~S}$

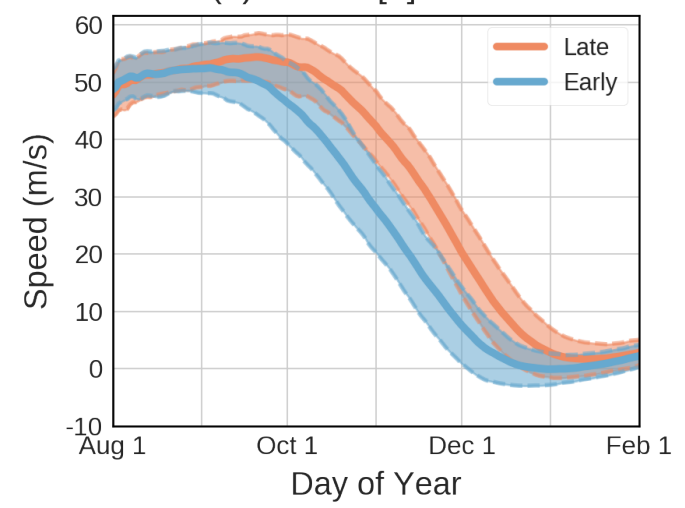

(b) $850 \mathrm{hPa}[\mathrm{u}] 55-65 \mathrm{~S}$

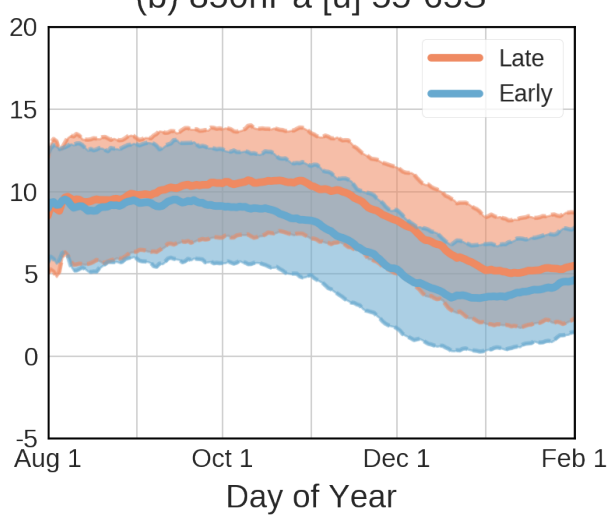

Figure 11. (a) Mean (solid line) and mean plus/minus one standard deviation (dashed lines) for $50 \mathrm{hPa}[\mathrm{u}] 55-65 \mathrm{~S}$ for late (red) and early (blue) years in hindcast ensemble for August 1 initialisations. (b) Similar, but for $850 \mathrm{hPa}$ [u] 55-65S. See text for details on how early and late years are defined. The year of 2002 is excluded from all calculations. 
(a) $50 \mathrm{hPa}[\mathrm{u}] 55-65 \mathrm{~S}$

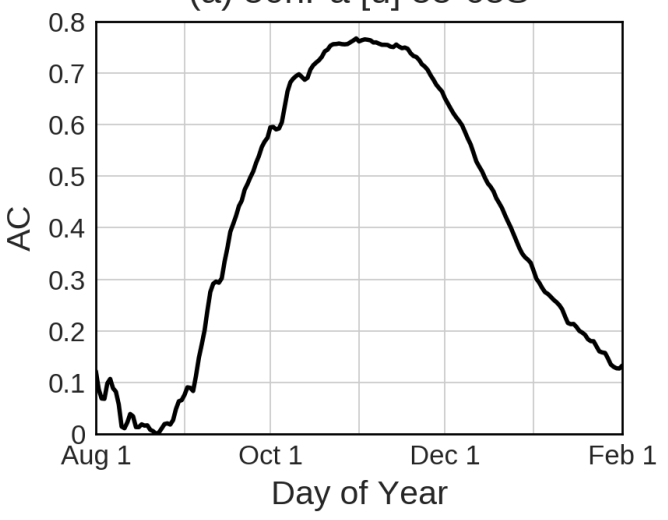

(b) $850 \mathrm{hPa}$ [u] $55-65 \mathrm{~S}$

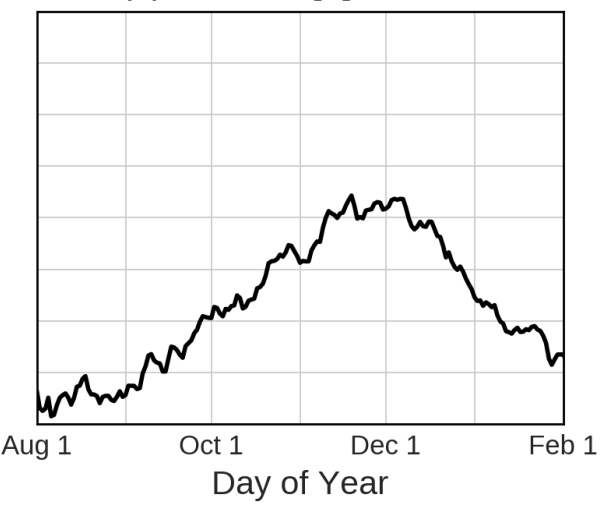

Figure 12. Expected AC for two-state model for (a) $50 \mathrm{hPa}[\mathrm{u}] 55-65 \mathrm{~S}$ and (b) $850 \mathrm{hPa}[\mathrm{u}]$ 55-65S.

a perfect forecast model, $\mathrm{AC}$ values for a given verification time series will still likely differ from this expected $\mathrm{AC}$ value due to sampling effects associated with finite ensemble and verification time series length.

The expected anomaly correlation for the two-state model is shown in Figure 12. In both the stratosphere and troposphere this value is seen to increase monontonically from about September until November as a result of an increase in the signal during this period (Figure 11). Maximum values in the troposphere emerge during November and are seen to persist into December. These values then rapidly decay from mid-December onwards, following the conclusion of the SPV breakdown event. The results of this simple model suggest that predictable shifts of the SPV seasonal cycle should lead to nonnegligible values of S2S forecast skill in the troposphere between mid-October and January.

We now consider whether there is agreement between the predictions of this twostate model and a more general signal-to-noise model. In this more general model the signal is defined as the interannual standard deviation of the ensemble mean and the noise is defined as the standard deviation of the ensemble members about the ensemble mean. 
The advantage of this model over the two-state model is that it allows for a more realistic representation of the signal - it recognises that forecast skill may be larger in years where there is a more extreme shift of the stratospheric seasonal cycle. More specifically, it allows for a continuous rather than a discrete representation of the signal. A potential disadvantage of this alternative signal-to-noise model is that recent work has suggested that some forecast models used in numerical weather prediction may be over-dispersive ([Scaife and Smith, 2018]; but see also Weisheimer et al. [2019]). If such an over-dispersive scenario was the case for the present hindcast ensemble, then this alternative signal-tonoise model would offer an unduly pessimistic estimate of S2S forecast skill. In particular, if the present hindcast ensemble were to be over-dispersive in its forecasts for shifts of the stratospheric seasonal cycle, then this would likely have a negative impact on estimates of S2S tropospheric forecast skill. In such a scenario, comparison with predictions from the two-state model may be instructive as it only predicts the sign of the shift of the stratospheric seasonal cycle, not its magnitude.

Tropospheric values for signal, noise and expected AC from the more general signalto-noise model are shown for the hindcasts initialised on August 1 and November 1 in Table 2 and Table 3. We have also included 5 and $95 \%$ confidence intervals for the expected AC by employing a bootstrap procedure over the hindcast ensemble; these confidence intervals quantify the sampling uncertainty associated with using finite (i.e. 34year) verification time series. To first order, the predictions of this more general signalto-noise model are in agreement with the predictions from the two-state model. In particular, the tropospheric signal is predicted to be largest between November and January in both of the methods and for both August 1 and November 1 initialisations. To further assess the predictions of both of these signal-to-noise models, we compute $\mathrm{AC}$ values between ERA-Interim and the ensemble means of hindcasts initialised on both August 1 and November 1 (Figures 13a and 13b). Prior to computing these AC values, we first apply a running-mean to all data. The length of the running-mean used for each figure is motivated by the forecast lead time (31-day and 7-day running means respectively) as this method has previously been suggested as appropriate for verifiying forecasts on S2S timescales [White et al., 2017, see their Figure 1]. The AC values in Figures $13 \mathrm{a}$ and $13 \mathrm{~b}$ are found to agree well with the predictions from both the two-state model and the more general signal-to-noise model, in terms of both amplitude and seasonality. This agreement is particularly striking for the August 1 initialisations, where 


\begin{tabular}{|c|c|c|c|c|c|c|}
\hline Month & Signal (S) & Noise $(\mathrm{N})$ & $\mathrm{AC}-\mathrm{S} / \mathrm{N}$ & AC - ERA & RMSE/Spread & RMSE/SD-ERA \\
\hline Aug & 1.51 & 1.40 & $0.73(0.59,0.84)$ & 0.78 & $1.36(0.83,1.26)$ & 0.66 \\
\hline Sep & 0.57 & 1.86 & $0.29(-0.06,0.49)$ & -0.12 & $0.98(0.81,1.28)$ & 1.09 \\
\hline Oct & 0.59 & 1.93 & $0.29(-0.04,0.50)$ & 0.23 & $1.23(0.83,1.25)$ & 0.98 \\
\hline Nov & 0.77 & 2.29 & $0.32(-0.01,0.52)$ & 0.45 & $1.39(0.82,1.28)$ & 0.90 \\
\hline Dec & 0.84 & 2.37 & $0.33(0.02,0.53)$ & 0.30 & $1.08(0.83,1.25)$ & 0.95 \\
\hline Jan & 0.70 & 2.03 & $0.32(0.01,0.52)$ & 0.28 & $1.11(0.83,1.25)$ & 0.96 \\
\hline
\end{tabular}

Table 2. Values of signal (m/s), noise (m/s), expected AC (along with $5 \%-95 \%$ confidence interval), ERA-Interim AC, RMSE/Spread (along with 5\% - 95\% confidence interval) and RMSE/SD(ERA) for monthly-mean [u] 55-65S, 850hPa for August 1 initialisation. Please see text for details on how all of these quantities are defined. The year of 2002 is excluded from all calculations. Bold values for AC - ERA indicate quantities that are statistically different from zero at the $5 \%$ level based on a two-sided two-sample t-test.

\begin{tabular}{||ccccccc}
\hline Month & Signal (S) & Noise (N) & AC - S/N & AC - ERA & RMSE/Spread & RMSE/SD-ERA \\
\hline \hline Nov & 1.45 & 1.34 & $0.74(0.62,0.83)$ & $\mathbf{0 . 7 1}$ & $1.03(0.83,1.26)$ & 0.71 \\
\hline Dec & 1.12 & 2.12 & $0.47(0.20,0.65)$ & $\mathbf{0 . 4 6}$ & $1.04(0.83,1.26)$ & 0.89 \\
\hline Jan & 0.71 & 1.96 & $0.34(0.02,0.55)$ & $\mathbf{0 . 3 7}$ & $1.12(0.83,1.26)$ & 0.93 \\
\hline
\end{tabular}

Table 3. As in Table 2, but for November 1 initialisation. Please note that hindcast AC values in this table are not directly comparable with those in Figure 13b as here monthly means are used rather than 7 -day means. 
(a) August 1

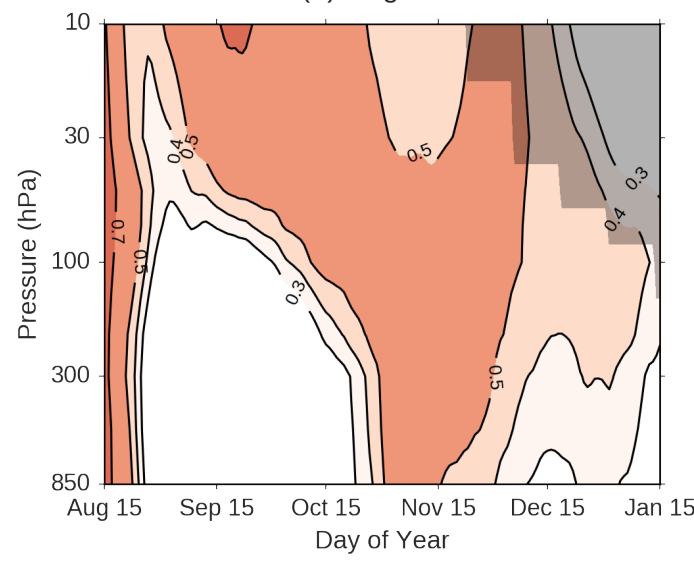

[u] 55-65S Hindcast Correlation

(b) November 1

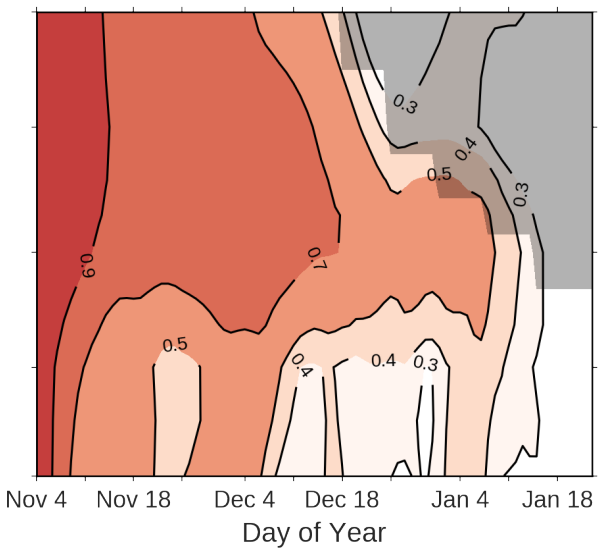

Figure 13. (a) Correlation between 31-day mean ensemble-mean [u] 55-65S and 31-day mean [u] 55-65S in ERA-Interim as a function of calendar day and pressure level for August 1 initialisation. Values on x-axis represent central date of 31-day mean. (b) As in (a), but for 7-day means for November 1 initialisation; note the expanded horizontal scale. In both figures all filled contour regions are statistically significant at the $5 \%$ level based on a two-sided two-sample t-test. Shaded area in top-right corner of each plot represents region where variability of [u] 55$65 \mathrm{~S}$ becomes very small following SPV breakdown event. The year of 2002 is excluded from all calculations. Please note non-linear scale for contour intervals in the stratosphere. 
tropospheric skill is seen to vanish following initialisation only to re-emerge again from October onwards. A similar result was previously found using a different set of hindcasts in Seviour et al. [2014]. As in that study, there is no evidence of a 'signal-to-noise' paradox, as all $\mathrm{AC}$ values are seen to fall within the 5-95\% confidence interval of the expected AC from the hindcasts (Table 2 and Table 3 ).

As an alternative test of these conclusions, we compute the root-mean-square error of the ensemble-mean forecast (RMSE) and consider its ratio with the hindcast ensemble standard deviation about the ensemble mean (RMSE/Spread) and with the ERAInterim interannual standard deviation (RMSE/SD-ERA; Table 2 and Table 3). For RMSE/Spread, a value less than one is an indicator of an over-dispersive hindcast ensemble and a value greater than one is an indicator of an under-dispersive ensemble. We have also included confidence intervals for RMSE/Spread; these were produced in a similar way to the confidence intervals for expected AC. Inspection of the results leads to the same conclusion as before: there is no evidence of an over-dispersive model ensemble (i.e., a 'signal-tonoise' paradox) for all months considered. Under these conditions, and under the assumptions of the general signal-to-noise model, the expected ratio RMSE/SD-ERA can be shown to equal $\frac{1}{\left(1+s^{2}\right)^{0.5}}$, where $s$ is the signal-to-noise ratio. Hence the smaller the value, the more predictable is the state. We can see from Table 2 that there is evidence of a re-emergence of skill from October in the August 1 initialisations. Thus we conclude that there is evidence for tropospheric predictability on S2S timescales in austral spring and summer, and that there is no evidence of a signal-to-noise paradox between the hindcasts and reanalysis during this time.

Before summarising our results, we note that skilful forecasts of the EDJ may also indirectly act as a source of skill for other components of the climate system. As a particular example we highlight Antarctic sea-ice extent (Figure 14). During years where there is an early equatorward transition of the EDJ, summertime Antarctic sea-ice is seen to retreat more rapidly, with the opposite behaviour during years where there is a delay in the transition. It may also be possible to use such forecasts to infer behaviour about autumn Antarctic sea-ice extent, based on persistence of summertime SSTs [Doddridge and Marshall, 2018]. However, it should be cautioned that the seasonal retreat of Antarctic sea-ice is not restricted to the month of November alone [e.g., Turner et al., 2017], and that November forecasts of the EDJ may only offer, at best, partial predictive power for summer and autumn Antarctic sea-ice extent. 


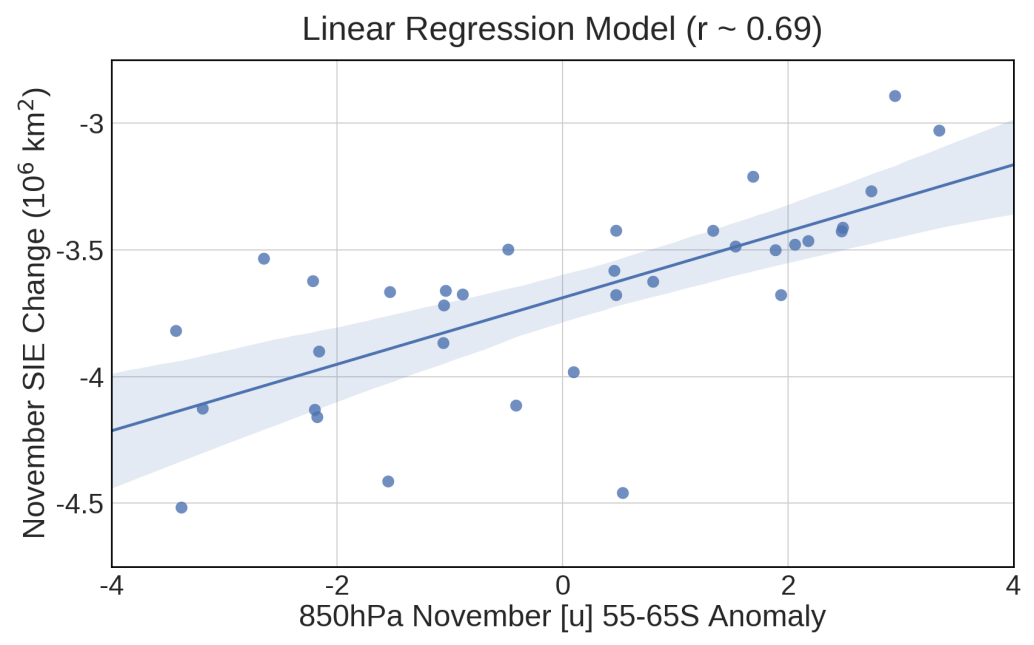

Figure 14. Linear regression between $850 \mathrm{hPa}$ November $[\mathrm{u}] 55-65 \mathrm{~S}$ anomaly $(\mathrm{m} / \mathrm{s})$ and November change in Antarctic sea-ice extent $\left(\mathrm{km}^{2}\right)$ from ERA-Interim and NSIDC, 1981-2015 (see Data and Methods section for further details). Shaded region represents $95 \%$ confidence interval for regression line. The year of 2002 is excluded from the calculation.

\section{Summary and Discussion}

In this paper we have addressed specific issues of sampling variability and S2S predictability for the SH extratropical atmosphere by analysing a large ensemble of hindcasts. Firstly, we have considered the impact of sampling variability on previous reanalysisbased results for the relationship between the SPV, EDJ and ENSO. We have found that coupled variability between the SPV and EDJ in the hindcast ensemble is in good agreement with the reanalysis, and that this coupled variability is robust to sampling effects. This coupled relationship between the SPV and the EDJ can be parsimoniously viewed as a continuous shift of the entire seasonal cycle during austral spring and summer [Byrne and Shepherd, 2018]. Moreover, the large sample size of the hindcast ensemble allows the detection of nonlinearity in the SPV-EDJ relationship. We have also found that coupled variability between ENSO and EDJ is robust to sampling variability but appears to be via a stratospheric pathway, at least from August until the SPV breakdown event sometime in austral summer. It should be noted that this result relates only to the highlatitude zonally symmetric response to ENSO; it does not relate to any potential highlatitude zonally asymmetric response. 
Secondly, we have used the hindcast ensemble to show that shifts of the stratospheric seasonal cycle during this time of year can be expected to be predictable on S2S timescales, confirming what has been found in several previous studies. Following on from this result, we have introduced two statistical models of 'signal' and 'noise' for the troposphere, to estimate the predictable component of tropospheric variability associated with these predictable shifts of the stratospheric seasonal cycle. Both of these statistical models indicate that tropospheric predictability on S2S timescales is considerable between about mid-October and January. We have confirmed that the predictions of both of these signal/noise models are in good agreement with hindcasts that have been verified against a reanalysis. All of these results provide evidence of tropospheric predictability on S2S timescales from at least as early as August 1, and show no evidence of a 'signal-to-noise paradox' between the hindcasts and the reanalysis [Scaife and Smith, 2018]. We note that it may be the case that tropospheric predictability is larger in years with a more severe shift of the stratospheric seasonal cycle, with the SSW of 2002 perhaps the most extreme example of such behavior [see Thompson et al., 2005, for a discussion of tropospheric impacts associated with the SSW of 2002.].

A potential future extension of our results relates to the early and mid-winter behavior of the SPV, when the SPV undergoes a poleward shift as part of its seasonal cycle [Shiotani et al., 1993; Kuroda and Kodera, 1998]. The timing of this poleward shift is closely linked to the strength of the SPV during winter, and hence also to the strength of the SPV during spring and early summer [Hio and Yoden, 2005]. Thus it may be the case that skilful forecasts of the EDJ during spring and summer can be made from as early as June 1, based on knowledge of the timing of the poleward shift of the SPV during winter [Lim et al., 2018]. Implicit in this statement is the assumption that a forecast model contains a realistic representation of the SPV seasonal cycle; given the broad spectrum of sub-gridscale parametrisations currently in use, this may not always be the case [Polichtchouk et al., 2018].

\section{Acknowledgments}

The authors thank Tim Stockdale and three anonymous reviewers for their helpful discussion and constructive comments. We acknowledge the provision of seasonal hindcast data from System 4 and the reanalysis data ERA-Interim by ECMWF. We acknowledge the provision of sea ice data by the U.S. National Snow and Ice Data Center. The us- 
age of this data has been cited in the text and included within the reference list. Funding support is acknowledged from the European Research Council Advanced Grant 'Understanding the Atmospheric Circulation Response to Climate Change' (ACRCC), Project 339390.

\section{References}

Baldwin, M. P., and T. J. Dunkerton (2001), Stratospheric Harbingers of Anomalous Weather Regimes, Science, 294(5542), 581-584, doi:10.1126/science.1063315.

Black, R. X., and B. A. McDaniel (2007), Interannual variability in the Southern Hemisphere circulation organized by stratospheric final warming events, Journal of the Atmospheric Sciences, 64(8), 2968-2974, doi:10.1175/JAS3979.1.

Byrne, N. J., and T. G. Shepherd (2018), Seasonal Persistence of Circulation Anomalies in the Southern Hemisphere Stratosphere and Its Implications for the Troposphere, Journal of Climate, 31(9), 3467-3483, doi:10.1175/JCLI-D-170557.1.

Byrne, N. J., T. G. Shepherd, T. Woollings, and R. A. Plumb (2017), Nonstationarity in Southern Hemisphere climate variability associated with the seasonal breakdown of the stratospheric polar vortex, Journal of Climate, 30(18), 71257139, doi:10.1175/JCLI-D-17-0097.1.

Dee, D. P., S. M. Uppala, A. J. Simmons, P. Berrisford, P. Poli, S. Kobayashi, U. Andrae, M. A. Balmaseda, G. Balsamo, P. Bauer, P. Bechtold, A. C. M. Beljaars, L. van de Berg, J. Bidlot, N. Bormann, C. Delsol, R. Dragani, M. Fuentes, A. J. Geer, L. Haimberger, S. B. Healy, H. Hersbach, E. V. Hólm, L. Isaksen, P. Kållberg, M. Köhler, M. Matricardi, A. P. McNally, B. M. Monge-Sanz, J. J. Morcrette, B. K. Park, C. Peubey, P. de Rosnay, C. Tavolato, J. N. Thépaut, and F. Vitart (2011), The ERA-Interim reanalysis: Configuration and performance of the data assimilation system, Quarterly Journal of the Royal Meteorological Society, 137(656), 553-597, doi:10.1002/qj.828.

Doddridge, E. W., and J. Marshall (2018), Modulation of the Seasonal Cycle of Antarctic Sea Ice Extent Related to the Southern Annular Mode, Geophysical Research Letters, 44(19), 9761-9768.

Domeisen, D. I., C. I. Garfinkel, and A. H. Butler (2019), The Teleconnection of El Nino Southern Oscillation to the Stratosphere, Reviews of Geophysics, 57, 5-47, 
doi:10.1029/2018RG000596.

Gerber, E. P., M. P. Baldwin, H. Akiyoshi, J. Austin, S. Bekki, P. Braesicke, N. Butchart, M. Chipperfield, M. Dameris, S. Dhomse, S. M. Frith, R. R. Garcia, H. Garny, A. Gettelman, S. C. Hardiman, A. Karpechko, M. Marchand, O. Morgenstern, J. E. Nielsen, S. Pawson, T. Peter, D. A. Plummer, J. A. Pyle, E. Rozanov, J. F. Scinocca, T. G. Shepherd, and D. Smale (2010), Stratosphere-troposphere coupling and annular mode variability in chemistryclimate models, Journal of Geophysical Research: Atmospheres, 115(D00M06), doi:10.1029/2009JD013770.

Hio, Y., and S. Yoden (2005), Interannual variations of the seasonal march in the Southern Hemisphere stratosphere for 1979 - 2002 and characterization of the unprecedented year 2002, Journal of the Atmospheric Sciences, 62(3), 567-580, doi:10.1175/JAS-3333.1.

Hitchcock, P., and I. R. Simpson (2014), The Downward Influence of Stratospheric Sudden Warmings, Journal of the Atmospheric Sciences, 71(10), 3856-3876, doi: 10.1175/JAS-D-14-0012.1.

Hurwitz, M. M., P. A. Newman, L. D. Oman, and A. M. Molod (2011), Response of the Antarctic Stratosphere to Two Types of El Nino Events, Journal of the Atmospheric Sciences, 68(4), 812-822, doi:10.1175/2011JAS3606.1.

Kumar, A. (2009), Finite Samples and Uncertainty Estimates for Skill Measures for Seasonal Prediction, Monthly Weather Review, 137(8), 2622-2631.

Kuroda, Y., and K. Kodera (1998), Interannual variability in the troposphere and stratosphere of the Southern Hemisphere winter, Journal of Geophysical Research, 103(D12), 13,787, doi:10.1029/98JD01042.

L'Heureux, M. L., and D. W. J. Thompson (2006), Observed Relationships between the El Nino-Southern Oscillation and the Extratropical Zonal-Mean Circulation, Journal of Climate, 19(2), 276-287, doi:10.1175/JCLI3617.1.

Lim, E.-P., H. H. Hendon, and H. Rashid (2013), Seasonal Predictability of the Southern Annular Mode due to Its Association with ENSO, Journal of Climate, $26(20), 8037-8054$.

Lim, E.-P., H. H. Hendon, and D. W. J. Thompson (2018), Seasonal Evolution of Stratosphere-Troposphere Coupling in the Southern Hemisphere and Implications for the Predictability of Surface Climate, Journal of Geophysical Research: 

Atmospheres, 123(21), 12,002-12,016, doi:10.1029/2018JD029321.

Lin, P., Q. Fu, and D. L. Hartmann (2012), Impact of Tropical SST on Stratospheric Planetary Waves in the Southern Hemisphere, Journal of Climate, 25(14), 50305046, doi:10.1175/JCLI-D-11-00378.1.

Molteni, F., T. Stockdale, M. A. Balmaseda, G. Balsamo, R. Buizza, L. Ferranti, L. Magnusson, K. Mogensen, T. N. Palmer, and F. Vitart (2011), The new ECMWF seasonal forecast system (System 4), 656, ECMWF, Reading, UK.

Polichtchouk, I., T. G. Shepherd, and N. J. Byrne (2018), Impact of Parametrized Nonorographic Gravity Wave Drag on Stratosphere-Troposphere Coupling in the Northern and Southern Hemispheres, Geophysical Research Letters, 45(16), 8612-8618, doi:10.1029/2018GL078981.

Polvani, L. M., L. Sun, A. H. Butler, J. H. Richter, and C. Deser (2017), Distinguishing Stratospheric Sudden Warmings from ENSO as Key Drivers of Wintertime Climate Variability over the North Atlantic and Eurasia, Journal of Climate, 30 (6), 1959-1969, doi:10.1175/JCLI-D-16-0277.1.

Roff, G., D. W. J. Thompson, and H. Hendon (2011), Does increasing model stratospheric resolution improve extended-range forecast skill?, Geophysical Research Letters, 38(5), doi:10.1029/2010GL046515.

Roscoe, H. K., J. D. Shanklin, and S. R. Colwell (2005), Has the Antarctic vortex split before 2002?, Journal of the Atmospheric Sciences, 62(3), 581-588, doi: 10.1175/JAS-3331.1.

Scaife, A. A., and D. Smith (2018), A signal-to-noise paradox in climate science, npj Climate and Atmospheric Science, 1(1), 28, doi:10.1038/s41612-018-0038-4.

Seager, R., N. Harnik, Y. Kushnir, W. Robinson, and J. Miller (2003), Mechanisms of Hemispherically Symmetric Climate Variability, Journal of Climate, 16(18), 2960-2978, doi:10.1175/1520-0442(2003)016;2960:MOHSCV ¿2.0.CO;2.

Seviour, W. J. M., S. C. Hardiman, L. J. Gray, N. Butchart, C. MacLachlan, and A. A. Scaife (2014), Skillful seasonal prediction of the Southern Annular Mode and Antarctic ozone, Journal of Climate, 27(19), 7462-7474, doi:10.1175/JCLI-D14-00264.1.

Shiotani, M., N. Shimoda, and I. Hirota (1993), Interannual variability of the stratospheric circulation in the Southern Hemisphere, Quarterly Journal of the Royal Meteorological Society, 119(511), 531-546. 
Sigmond, M., J. F. Scinocca, V. V. Kharin, and T. G. Shepherd (2013), Enhanced seasonal forecast skill following stratospheric sudden warmings, Nature Geoscience, 6, 98-102, doi:10.1038/ngeo1698.

Son, S. W., A. Purich, H. H. Hendon, B. M. Kim, and L. M. Polvani (2013), Improved seasonal forecast using ozone hole variability?, Geophysical Research Letters, 40(23), 6231-6235, doi:10.1002/2013GL057731.

Son, S.-W., B.-R. Han, C. Garfinkel, S.-Y. Kim, R. Park, N. Abraham, H. Akiyoshi, A. Archibald, N. Butchart, M. Chipperfield, M. Dameris, M. Deushi, S. Dhomse, S. Hardiman, P. Joeckel, D. Kinnison, M. Michou, O. Morgenstern, F. O'Connor, L. Oman, D. Plummer, A. Pozzer, L. Revell, E. Rozanov, A. Stenke, K. Stone, S. Tilmes, Y. Yamashita, and G. Zeng (2018), Tropospheric jet response to Antarctic ozone depletion: An update with Chemistry-Climate Model Initiative (CCMI) models, Environmental Research Letters, 13(5).

Sun, L., G. Chen, and W. A. Robinson (2014), The role of stratospheric polar vortex breakdown in Southern Hemisphere climate trends, Journal of the Atmospheric Sciences, 71 (7), 2335-2353, doi:10.1175/JAS-D-13-0290.1.

Thompson, D. W., M. Baldwin, and S. Solomon (2005), Stratosphere-troposphere coupling in the Southern Hemisphere, Journal of Atmospheric Sciences, 62, 708715 .

Turner, J., T. Phillips, G. J. Marshall, J. S. Hosking, J. O. Pope, T. J. Bracegirdle, and P. Deb (2017), Unprecedented springtime retreat of Antarctic sea ice in 2016, Geophysical Research Letters, 44(13), 6868-6875.

van Loon, H. (1967), The half-yearly oscillations in middle and high southern latitudes and the coreless winter, Journal of the Atmospheric Sciences, 24, 472-486, doi:10.1175/1520-0469(1967)024¡0472:THYOIM ¿2.0.CO;2.

Vera, C. S., and M. Osman (2018), Activity of the Southern Annular Mode during 2015-2016 El Nino event and its impact on Southern Hemisphere climate anomalies, International Journal of Climatology, 38(S1), 1288-1295.

Weisheimer, A., D. Decremer, D. MacLeod, C. O'Reilly, T. Stockdale, S. Johnson, and T. Palmer (2019), How confident are predictability estimates of the winter North Atlantic Oscillation?, Quarterly Journal of the Royal Meteorological Society, $O(0)$, doi:10.1002/qj.3446. 
White, C. J., H. Carlsen, A. W. Robertson, R. J. Klein, J. K. Lazo, A. Kumar, F. Vitart, E. Coughlan de Perez, A. J. Ray, V. Murray, S. Bharwani, D. MacLeod, R. James, L. Fleming, A. P. Morse, B. Eggen, R. Graham, E. Kjellström, E. Becker, K. V. Pegion, N. J. Holbrook, D. McEvoy, M. Depledge, S. PerkinsKirkpatrick, T. J. Brown, R. Street, L. Jones, T. A. Remenyi, I. HodgsonJohnston, C. Buontempo, R. Lamb, H. Meinke, B. Arheimer, and S. E. Zebiak (2017), Potential applications of subseasonal-to-seasonal (S2S) predictions, Meteorological Applications, $24(3), 315-325$.

WMO (2013), Subseasonal-to-seasonal prediction: research implementation plan, Tech. rep., WMO, https://library.wmo.int/pmb_ged/2014_wcrp_subseasonal_to_seasonal_prediction_en.pdf.

Zubiaurre, I., and N. Calvo (2012), The El Nino-Southern Oscillation (ENSO) Modoki signal in the stratosphere, Journal of Geophysical Research: Atmospheres, 117 (D4), doi:10.1029/2011JD016690. 